\title{
World-Universe Model-Alternative to Big Bang Model
}

\author{
Vladimir S. Netchitailo \\ Biolase Inc., Cromwell, CA, USA \\ Email:netchitailov@gmail.com
}

How to cite this paper: Netchitailo, V.S. (2020) World-Universe Model-Alternative to Big Bang Model. Journal of High Energy Physics, Gravitation and Cosmology, 6, 133-158.

https://doi.org/10.4236/jhepgc.2020.61012

Received: December 10, 2019

Accepted: January 17, 2020

Published: January 20, 2020

Copyright $\odot 2020$ by author(s) and Scientific Research Publishing Inc. This work is licensed under the Creative Commons Attribution International License (CC BY 4.0).

http://creativecommons.org/licenses/by/4.0/ (c) (i) Open Access

\begin{abstract}
This manuscript provides a comparison of the Hypersphere World-Universe Model (WUM) with the prevailing Big Bang Model (BBM) of the Standard Cosmology. The performed analysis of BBM shows that the Four Pillars of the Standard Cosmology are model-dependent and not strong enough to support the model. The angular momentum problem is one of the most critical problems in BBM. Standard Cosmology cannot explain how Galaxies and Extra Solar systems obtained their substantial orbital and rotational angular momenta, and why the orbital momentum of Jupiter is considerably larger than the rotational momentum of the Sun. WUM is the only cosmological model in existence that is consistent with the Law of Conservation of Angular Momentum. To be consistent with this Fundamental Law, WUM discusses in detail the Beginning of the World. The Model introduces Dark Epoch (spanning from the Beginning of the World for 0.4 billion years) when only Dark Matter Particles (DMPs) existed, and Luminous Epoch (ever since for 13.8 billion years). Big Bang discussed in Standard Cosmology is, in our view, transition from Dark Epoch to Luminous Epoch due to Rotational Fission of Overspinning Dark Matter (DM) Supercluster's Cores. WUM envisions Matter carried from the Universe into the World from the fourth spatial dimension by DMPs. Ordinary Matter is a byproduct of DM annihilation. WUM solves a number of physical problems in contemporary Cosmology and Astrophysics through DMPs and their interactions: Angular Momentum problem in birth and subsequent evolution of Galaxies and Extrasolar systems-how do they obtain it; Fermi Bubbles-two large structures in gamma-rays and X-rays above and below Galactic center; Diversity of Gravitationally-Rounded Objects in Solar system; some problems in Solar and Geophysics [1]. WUM reveals Inter-Connectivity of Primary Cosmological Parameters and calculates their values, which are in good agreement with the latest results of their measurements.
\end{abstract}




\section{Keywords}

Big Bang Model, Four Pillars of Standard Cosmology, Angular Momentum Problem, Black Holes, Hypersphere World-Universe Model, Multicomponent Dark Matter, Macroobjects Structure, Law of Conservation of Angular Momentum, Medium of the World, Inter-Connectivity of Primary Cosmological Parameters, The Beginning of the World, Dark Epoch, Rotational Fission, Luminous Epoch, Macroobject Shell Model, Dark Matter Core, Gravitational Burst, Intergalactic Plasma, Microwave Background Radiation, Far-Infrared Background Radiation, Emergent Phenomena, CODATA

We can't solve problems by using the same kind of thinking we used when we created them.

Albert Einstein

\section{Introduction}

Hypersphere World-Universe Model (WUM) is proposed as an alternative to the prevailing Big Bang Model of Standard Cosmology. WUM is a classical model, and is described by classical notions, which define emergent phenomena. By definition, an emergent phenomenon is a property that is a result of simple interactions that work cooperatively to create a more complex interaction. Physically, simple interactions occur at a microscopic level, and the collective result can be observed at a macroscopic level. WUM introduces classical notions once the very first ensemble of particles has been created at the cosmological time $\cong 10^{-18} \mathrm{~s}$ (state of the World at cosmological times $<10^{-18} \mathrm{~s}$ is best described by Quantum Mechanics).

WUM is based on two parameters: dimensionless Rydberg constant $\alpha=\left(2 a R_{\infty}\right)^{1 / 3}$, where $R_{\infty}$ is Rydberg constant, $a$ is the basic unit of size (classical electron radius equals to: $\left.a_{o}=a / 2 \pi\right)$; and a dimensionless time-varying parameter $Q$, which is a measure of the Size $R$ and Age $A_{\tau}$ of the World $Q=R / a=A_{\tau} / t_{0}$, where $t_{0}=a / c$ is the basic unit of time and $c$ is the gravitodynamic constant. In the present Epoch, $Q=0.759972 \times 10^{40}$. It is worth to note that the constant $\alpha$ was later named "Sommerfeld's constant," and subsequently "Fine-structure constant" [1].

The Big Bang Model (BBM) offers a comprehensive explanation for a broad range of observed phenomena. The framework for the BBM relies on General Relativity and on simplifying assumptions such as homogeneity and isotropy of space. The Lambda Cold Dark Matter $(\Lambda \mathrm{CDM})$ model is a parametrization of the BBM in which the universe contains three major components: first, a Cosmological constant $\Lambda$ associated with dark energy; second, the postulated Cold Dark Matter (CDM); and third, Ordinary matter.

The $\Lambda$ CDM model is based on six parameters: baryon density $\Omega_{B}$, dark mat- 
ter density $\Omega_{D M}$, dark energy density $\Omega_{\Lambda}$, scalar spectral index, curvature fluctuation amplitude, and reionization optical depth. The values of these six parameters are mostly not predicted by current theory; other possible parameters are fixed at "natural" values e.g. total density equals to 1.00, neutrino masses are small enough to be negligible. The $\Lambda$ CDM model can be extended by adding cosmological inflation. It is frequently referred to as the Standard Model of Big Bang (BB) cosmology, which is the classical model too.

The Four Pillars of the Standard Cosmology are as follows [2]:

- Expansion of the Universe;

- Origin of the cosmic background radiation;

- Nucleosynthesis of the light elements;

- Formation of galaxies and large-scale structures.

BBM and WUM are principally different models. Comparison of the main parameters of the models is presented in Table 1.

Angular momentum problem is one of the most critical problems in the Standard Cosmology that must be solved. Any theory of evolution of the Universe that is not consistent with the Law of Conservation of Angular Momentum should be promptly ruled out. To the best of our knowledge, the Hypersphere World-Universe Model is the only cosmological model in existence that is consistent with this Fundamental Law (see Sections 3.7, 3.8 and 3.9).

\section{Analysis of the Big Bang Model}

\subsection{Expansion of the Universe}

The fact that galaxies are receding from us in all directions was first discovered

Table 1. Parameters of big bang model and world-universe model.

\begin{tabular}{|c|c|c|}
\hline Parameter & Big Bang Model & World-Universe Model \\
\hline Structure of the World & $3+1$ Spacetime & 3D Hypersphere of $4 \mathrm{D}$ Nucleus of the World. Time is a Factor of the World \\
\hline The Beginning & Singularity & $\begin{array}{l}\text { 4D Nucleus of the World with an extrapolated radius } a \text { as the result of a } \\
\text { fluctuation in the Universe }\end{array}$ \\
\hline Expansion & $\begin{array}{l}\text { Inflation-extremely rapid } \\
\text { exponential expansion of space }\end{array}$ & The radius of the Nucleus of the World is increasing with speed $c$ \\
\hline Content & $\begin{array}{l}\text { Dark Energy, Cold Dark Matter, } \\
\text { Ordinary matter }\end{array}$ & Multicomponent Dark Matter (DM), Ordinary matter \\
\hline Origin of Matter & Singularity & $\begin{array}{l}\text { DM comes from the Universe to the Nucleus along the fourth spatial } \\
\text { dimension. Ordinary Matter is a byproduct of DM annihilation }\end{array}$ \\
\hline Cosmic Microwave Background & $\begin{array}{l}\text { Photons wavelength is } \\
\text { increasing over time }\end{array}$ & Thermodynamic equilibrium of photons with Intergalactic plasma \\
\hline Nucleosynthesis of Light Elements & Big Bang Nucleosynthesis & $\begin{array}{l}\text { Nucleosynthesis of all elements (including light elements) occurs inside of } \\
\text { DM Cores of Macroobjects }\end{array}$ \\
\hline Primary Cosmological Parameters & Independent & Inter-connected \\
\hline Galactic Center & Black Hole & DM Core of Galaxy \\
\hline $\begin{array}{l}\text { Law of Conservation of Angular } \\
\text { Momentum }\end{array}$ & Inconsistent & Consistent \\
\hline
\end{tabular}


by Hubble. There is now excellent evidence for Hubble's law which states that the recessional velocity $v$ of a galaxy is proportional to its distance $d$ from us, that is, $v=H d$ where $H$ is Hubble's constant. Projecting galaxy trajectories backwards in time means that they converge to the cosmological Singularity at $t=0$ that is an infinite energy density state. This uncovers one of the shortcomings of the Standard Cosmology-the Horizon problem [3]: Why does the universe look the same in all directions when it arises out of causally disconnected regions? This problem is most acute for the very smooth cosmic microwave background radiation.

This problem was resolved by the cosmological Inflation, which is a theory of an extremely rapid exponential expansion of space in the early universe. This rapid expansion increased the linear dimensions of the early universe by a factor of at least $10^{26}$, and so increased its volume by a factor of at least $10^{78}$. The inflationary epoch lasted from $10^{-36} \mathrm{~s}$ after the conjectured $\mathrm{BB}$ singularity to some time between $10^{-33}$ and $10^{-32} \mathrm{~s}$ after the singularity. Following the inflationary period, the universe continued to expand, but at a slower rate.

"It's a beautiful theory, said Peebles. Many people think it's so beautiful that it's surely right. But the evidence of it is very sparse" [4].

According to Silk, our best theory of the beginning of the universe, inflation, awaits a definitive and falsifiable probe, in order to satisfy most physicists that it is a trustworthy theory. Our basic problem is that we cannot prove the theory of inflation is correct, but we urgently need to understand whether it actually occurred [5].

E. Conover in the paper "Debate over the universe's expansion rate may unravel physics. Is it a crisis?" outlined the following situation with the measurements of an expansion rate of the universe [6]:

- Scientists with the Planck experiment have estimated that the universe is expanding at a rate of $67.4 \mathrm{~km} / \mathrm{s} M p c$ with an experimental error of $0.5 \mathrm{~km} / \mathrm{s}$ $M p c$;

- But supernova measurements have settled on a larger expansion rate of 74.0

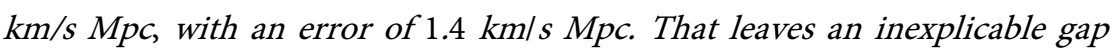
between the two estimates. Now "the community has started to take this [problem] extremely seriously", says cosmologist Daniel Scolnic of Duke University, who works on the supernova project led by Riess, called SHOES;

- It's unlikely that an experimental error in the Planck measurement could explain the discrepancy. That prospect is "not a possible route out of our current crisis," said cosmologist Lloyd Knox of the University of California, Davis;

- So, worries have centered on the possibility that the supernova measurements contain unaccounted for systematic errors-biases that push the SHOES estimate to larger value.

L. Verde, T. Treu, and A. G. Riess gave a brief summary of the "Workshop at Kavli Institute for Theoretical Physics, July 2019” [7]. It is not yet clear whether 
the discrepancy in the observations is due to systematics, or indeed constitutes a major problem for the Standard model.

\subsection{Origin of the Cosmic Background Radiation}

According to BBM, about 380,000 years after the Big Bang the temperature of the universe fell to the point where nuclei could combine with electrons to create neutral atoms. As a result, photons no longer interacted frequently with matter, the universe became transparent, and the Cosmic Microwave Background (CMB) radiation was created. This cosmic event is usually referred to as Decoupling. The photons that existed at the time of photon decoupling have been propagating ever since, though growing fainter and less energetic, since the expansion of space causes their wavelength to increase over time. The photons present at the time of decoupling are the same photons that we see in the CMB radiation now. But then, why the CMB is a perfect black-body?

According to WUM, wavelength is a classical notion. Photons, which are quantum objects, have only four-momenta. They don't have wavelengths. By definition, "Black-body radiation is the thermal electromagnetic radiation within or surrounding a body in thermodynamic equilibrium with its environment". In frames of WUM, the black-body spectrum of CMB is due to thermodynamic equilibrium of photons with the Intergalactic plasma [1], the existence of which is experimentally proved [8].

\subsection{Nucleosynthesis of the Light Elements}

Big Bang Nucleosynthesis (BBN) refers to the production of nuclei other than those of hydrogen during the early phases of the Universe. Primordial nucleosynthesis is believed to have taken place in the interval from roughly 10 seconds to 20 minutes after the Big Bang and is calculated to be responsible for the formation of most of the universe's helium as the isotope helium-4, along with small amounts of deuterium, helium-3, and a very small amount of lithium-7. Essentially all of the elements that are heavier than lithium were created much later, by stellar nucleosynthesis in evolving and exploding stars.

The history of BBN began with the calculations of R. Alpher in the 1940s. During the 1970s, there were major efforts to find processes that could produce deuterium, but those revealed ways of producing isotopes other than deuterium. The problem was that while the concentration of deuterium in the universe is consistent with the BBM as a whole, it is too high to be consistent with a model that presumes that most of the universe is composed of protons and neutrons. The standard explanation now used for the abundance of deuterium is that the universe does not consist mostly of baryons, but that non-baryonic dark matter makes up most of the mass of the universe [9].

According to modern cosmological theory, lithium was one of the three elements synthesized in the Big Bang. But in case of lithium, we observe a cosmological lithium discrepancy in the universe: older stars seem to have less lithium 
than they should, and some younger stars have much more. M. Anders, et al. report on the results of the first measurement of the ${ }^{2} \mathrm{H}(\alpha, \gamma)^{6} \mathrm{Li}$ cross section at Big Bang energies. The experiment was performed deep underground at the LUNA $400 \mathrm{kV}$ accelerator in Gran Sasso, Italy. A BBN lithium abundance ratio of ${ }^{6} \mathrm{Li} /{ }^{7} \mathrm{Li}=(1.5 \pm 0.3) \times 10^{-5}$ is obtained, firmly ruling out BBN lithium production as a possible explanation for the reported ${ }^{6} \mathrm{Li}$ detections [10].

In frames of WUM, Nucleosynthesis of all elements (including light elements) occurs inside of DM Cores of all Macroobjects during their evolution. The theory of Stellar Nucleosynthesis is well developed, starting with the publication of a celebrated $\mathrm{B}^{2} \mathrm{FH}$ review paper [11]. With respect to WUM, this theory should be expanded to include annihilation of heavy Dark Matter fermions in Macroobjects Cores [1].

\subsection{Formation of Galaxies and Large-Scale Structures}

The formation and evolution of galaxies can be explained only in terms of gravitation within an inflation + dark matter + dark energy scenario [12]. The standard Hot Big Bang model provides a framework for understanding galaxy formation. At about 10,000 years after the Big Bang, the temperature had fallen to such an extent that the energy density of the Universe began to be dominated by massive particles, rather than the light and other radiation which had predominated earlier. This change in the form of the main matter density meant that the gravitational forces between the massive particles could begin to take effects, so that any small perturbations in their density would grow.

This brings into focus one of the shortcomings of the Standard Cosmology-the density fluctuation problem [3]: The perturbations which gravitationally collapsed to form galaxies must have been primordial in origin; from whence did they arise?

There is another problem in the Standard Cosmology-angular momentum problem [1]:

- The Sun, for example, only accounts for about $0.3 \%$ of the total angular momentum of the Solar System (SS) while about $60 \%$ is attributed to Jupiter. Evolutionary theory cannot account for this. This strange distribution was the primary cause of the downfall of the Nebular hypothesis;

- SS has an orbital momentum $L_{o r b}^{S S}$ calculated based on the distance of 26.4 kly from the galactic center and orbital speed of about $220 \mathrm{~km} / \mathrm{s}$ :

$L_{\text {orb }}^{S S}=1.1 \times 10^{56} \mathrm{~J} \cdot \mathrm{s}$, which far exceeds rotational angular momentum:

$L_{\text {rot }}^{S S}=3.2 \times 10^{43} \mathrm{~J} \cdot \mathrm{s}$;

- Milky Way (MW) galaxy is gravitationally bounded with Local Supercluster (LS) and has an orbital momentum $L_{o r b}^{M W}$ calculated based on the distance of 65 million light-years from LS and orbital speed of about $400 \mathrm{~km} / \mathrm{s}$ [13]: $L_{\text {orb }}^{M W}=2.5 \times 10^{71} \mathrm{~J} \cdot \mathrm{s}$, which far exceeds the rotational angular momentum [14]: $L_{\text {rot }}^{M W} \approx 1 \times 10^{67} \mathrm{~J} \cdot \mathrm{s}$;

- How did MW galaxy and SS obtain their substantial orbital angular momenta? 
To the best of our knowledge, the Standard Model doesn't answer these questions. The Hypersphere World-Universe model is the only cosmological model in existence that is consistent with the Law of Conservation of Angular Momentum (see Sections 3.7, 3.8 and 3.9).

As a conclusion, the performed analysis shows that the Four Pillars of the Standard Cosmology are model-dependent and not strong enough to support the Big Bang model.

\subsection{Black Holes}

Black hole is a mathematical solution of Einstein's field equations for gravity in $3+$ 1 dimensional spacetime. The simplest black hole solution is the Schwarzschild solution, which describes the gravitational field in the spherically symmetric, static, vacuum case. This solution is characterized by a single parameter, which corresponds to the mass of an object that produces the same gravitational field [15].

The existence of supermassive objects in galactic centers is now commonly accepted. It is commonly believed that the central mass is a supermassive Black Hole (BH). There exists, however, evidence to the contrary. In late 2013, ICRAR astronomer Dr. N. Hurley-Walker spotted a previously unknown radio galaxy NGC1534 that is quite close to Earth at 248 million light-years but is much fainter than it should be if the central $\mathrm{BH}$ was accelerating the electrons in the jets: "The discovery is also intriguing because at some point in its history the central black hole switched off but the radio jets have persisted. This is a very rare occurrence-this is only the fifth of this type to be discovered, and by far the faintest. We can only see it at low frequencies, which tells us that the electrons in the jets are not getting new energy from the black hole, so it must have been switched off for some time. The interesting thing about the object I found is that it's being hosted by a spiral galaxy, like our own". It's also possible there was never a BH there at all [16].

Recently a population of large, very low surface brightness, spheroidal galaxies was identified in the Coma cluster. The apparent survival of these Ultra Diffuse Galaxies (UDGs) in a rich cluster suggests that they have very high masses. P. van Dokkum, et al. present the stellar kinematics of Dragonfly 44, one of the largest Coma UDGs, whose mass about equals that of the Milky Way. However, the galaxy emits only $1 \%$ of the light emitted by the Milky Way. Astronomers reported that this galaxy might be made almost entirely of dark matter. The existence of nearly-dark objects with this mass is unexpected, as galaxy formation is thought to be maximally-efficient in this regime [17].

Candidate stellar-mass BHs in globular clusters of the Milky Way were reported in the following publications:

- R. Narayan, J. E. McClintock, and I Yi present a new model for BH soft X-ray transients A0620-00, V404 Cyg, and X-ray Nova Mus 1991 with mass 4.4; 9 and 7 solar mass respectively [18];

- K. Gebhardt, R. M. Rich, and L. Ho present the detection of a 20,000 solar 
mass BH in the Stellar Cluster G1, which is one of the most massive stellar clusters in M31. In their opinion, Globular clusters in our Galaxy should be searched for central BHs [19];

- L. Chomiuk, et al. report the discovery of a candidate stellar-mass BH in the globular cluster M62, which they term M62-VLA1. The radio, X-ray, and optical properties of M62-VLA1 are very similar to those for V404 Cyg, one of the best-studied quiescent stellar-mass BHs [20];

- B. Giesers, et al. performed multiple epoch observations of NGC 3201 with the aim of constraining the binary fraction. The obtained data show strong evidence that the target star is in a binary system with a non-luminous object having a minimum mass of $(4.36 \pm 0.41)$ solar mass. This object should be degenerate, since it is invisible, and the minimum mass is significantly higher than the Chandrasekhar limit [21];

- J. Liu, et al. report radial velocity measurements of a Galactic star, LB-1 and find that the motion of it requires the presence of a dark companion with mass of $68_{-13}^{+11} M_{\odot}$. In author's opinion, forming such massive ones in a high-metallicity environment would be extremely challenging to current stellar evolution theories [22].

In 2014, L. Mersini-Houghton claimed to demonstrate mathematically that, given certain assumptions about $\mathrm{BH}$ firewalls, current theories of $\mathrm{BH}$ formation are flawed. She claimed that Hawking radiation causes the star to shed mass at a rate such that it no longer has the density sufficient to create a $\mathrm{BH}$ [23].

R. K. Leane and T. R. Slatyer in the paper "Revival of the Dark Matter Hypothesis for the Galactic Center Gamma-Ray Excess" examine the impact of unmodeled source populations on identifying the true origin of the galactic center $\mathrm{GeV}$ excess (GCE). The authors discover striking behavior consistent with a mismodeling effect in the real Fermi data, finding that large artificial injected dark matter signals are completely misattributed to point sources. Consequently, they conclude that dark matter may provide a dominant contribution to the GCE after all [24].

As a conclusion, we have the observational evidences for the existence of non-luminous objects in centers of galaxies (for example, by the orbits of stars in the center of our galaxy), globular clusters, binary systems, and commonly accept them as BHs. But they might be "Dark Stars" composed of fermion Dark Matter Particles. A mechanism whereby Dark Matter (DM) in protostellar halos plays a role in the formation of the first stars is discussed by D. Spolyar, K. Freese, and P. Gondolo [25]. Heat from neutralino DM annihilation is shown to overwhelm any cooling mechanism, consequently impeding the star formation process. A “dark star" powered by DM annihilation instead of nuclear fusion may result. Dark stars are in hydrostatic and thermal equilibrium, but with an unusual power source [26].

In frames of WUM, supermassive objects in galactic centers are Macroobjects with Cores made up of DM fermions (see Sections 3.1 and 3.2). 


\section{Hypersphere World-Universe Model}

There exist a number of competing cosmological models. In our opinion, the most probable model is the one that built on the minimum number of parameters. World-Universe Model (WUM) is based on two parameters only: dimensionless Rydberg constant $\alpha$ and dimensionless quantity $Q$, which increases in time $Q \propto \tau$, and is, in fact, a measure of the Size and Age of the World. In WUM we often use well-known physical parameters, keeping in mind that all of them can be expressed through the Basic Units. Taking the relative values of physical parameters in terms of the Basic Units we can express all dimensionless parameters of the World through two Fundamental Parameters $\alpha$ and $Q$ in various rational exponents, as well as small integer numbers and $\pi$.

Key concepts and observations of WUM are the followings [1]:

- The Beginning of the World;

- Expansion and Creation of Matter;

- Content of the World;

- Structure of Macroobjects;

- Inter-Connectivity of Primary Cosmological Parameters;

- Gravity, Space and Time are all emergent phenomena.

WUM makes reasonable assumptions in each of these areas. The remarkable agreement of the calculated values of the primary cosmological parameters with the observational data gives us considerable confidence in the Model (see Table 3, Section 3.6).

In WUM we introduce a basic unit of mass $m_{0}$ that equals to: $m_{0}=h / a c=70.025267 \mathrm{MeV}$, where $h$ is Planck constant. $m_{0}$ plays a key role when masses of DMPs are discussed below.

\subsection{Multicomponent Dark Matter}

Possibility of DMP observation in Centers of Macroobjects has drawn many new researchers to the field in the last forty years. Indirect effects in cosmic rays and gamma-ray background from the annihilation of cold DM in the form of heavy stable neutral leptons in Galaxies were considered in pioneer articles [27]-[32]. Important cosmological problems like Dark Matter and Dark Energy could be, in principle, solved through extended gravity. This is stressed, for example, in the famous paper of Prof. C. Corda [33]. A paper by G. Bertone and T. M. P. Tait [34] provides an excellent review of what we have learned about the nature of DM from past experiments, and the implications for planned DM searches in the next decade.

Two-component DM system consisting of bosonic and fermionic components is proposed for the explanation of emission lines from the bulge of Milky Way galaxy. C. Boehm, P. Fayet, and J. Silk analyze the possibility of two coannihilating neutral and stable DMPs: a heavy fermion (for example the lightest neutralino $>100 \mathrm{GeV})$ and a light spin-0 particle ( 100 MeV) [35]. 
In WUM, the World consists of DM (about 92.8\% of the total Matter) and Ordinary matter (about 7.2\%). It means that DM should play the main role in any Cosmological model. It is the case in WUM, and Ordinary matter is a byproduct of DMPs annihilation [1].

WUM proposes multicomponent DM system consisting of two couples of coannihilating DMPs: a heavy DM fermion-DMF1 (1.3 TeV) and a light spin-0 boson-DIRAC $(70 \mathrm{MeV})$ that is a dipole of Dirac's monopoles; a heavy fermion-DMF2 $(9.6 \mathrm{GeV})$ and a light spin-0 boson-ELOP $(340 \mathrm{keV})$ that is a dipole of preons; fermions-DMF3 (3.7 keV) and DMF4 (0.2 eV).

WUM postulates that masses of DMFs and bosons are proportional to $m_{0}$ multiplied by different exponents of $\alpha$ and can be expressed with the following formulae [1]:

DMF1 (fermion): $m_{\mathrm{DMF} 1}=\alpha^{-2} m_{0}=1.3149950 \mathrm{TeV}$;

DMF2 (fermion): $m_{\mathrm{DMF} 2}=\alpha^{-1} m_{0}=9.5959823 \mathrm{GeV}$;

DIRAC (boson): $m_{\text {DIRAC }}=\alpha^{0} m_{0}=70.025267 \mathrm{MeV}$;

ELOP (boson): $m_{\text {ELOP }}=2 / 3 \alpha^{1} m_{0}=340.66606 \mathrm{keV}$;

DMF3 (fermion): $m_{\mathrm{DMF} 3}=\alpha^{2} m_{0}=3.7289402 \mathrm{keV}$;

DMF4 (fermion): $m_{\mathrm{DMF} 4}=\alpha^{4} m_{0}=0.19857111 \mathrm{eV}$.

It is worth to note that Rydberg unit of energy $R y$ equals to: $R y=1 / 2 a^{3} m_{0} c^{2}=13.605693 \mathrm{eV}$.

The values of mass of DMF1, DMF2, DMF3 fall into the ranges estimated in literature for neutralinos, WIMPs, and sterile neutrinos respectively [1]. DMF1, DMF2 and DMF3 partake in the self-annihilation interaction with strength equals to $\alpha^{-2}, \alpha^{-1}$ and $\alpha^{2}$ respectively.

WUM introduces a new Weak Force, providing interaction between DMPs. The strength of this force $G_{W}$ is about 30 orders of magnitude greater than the strength of the gravity $G: G_{W}=G \times Q^{3 / 4}$, and its range in the present epoch $R_{W}=a \times Q^{1 / 4}=1.65314 \times 10^{-4} \mathrm{~m}$ is considerably greater than that of the weak nuclear force. Proposed Weak Interaction between DMPs provides the integrity of DM Cores of all Macroobjects [1].

\subsection{Macroobjects Cores Made Up of Dark Matter Particles}

According to WUM, Macrostructures of the World (Superclusters, Galaxies, Extrasolar Systems) have Nuclei made up of DMFs, which are surrounded by Shells composed of DM and Ordinary matter. The shells envelope one another, like a Russian doll. The lighter a particle, the greater the radius and mass of its shell. Innermost shells are the smallest and are made up of heaviest particles; outer shells are larger and consist of lighter particles [1].

Table 2 describes the parameters of Macroobjects Cores (which are Fermionic Compact Stars in WUM) in the present Epoch made up of different DM fermions: self-annihilating DMF1, DMF2, DMF3; fermion DMF4 and electron-positron plasma.

The calculated parameters of the shells show that [1]: 
Table 2. Parameters of Macroobject Cores made up of different Fermions in present Epoch.

\begin{tabular}{ccccc}
\hline Fermion & $\begin{array}{c}\text { Fermion } \\
\text { Mass } m_{f}, \mathrm{MeV}\end{array}$ & $\begin{array}{c}\text { Macroobject } \\
\text { Mass } M_{\max }, \mathrm{kg}\end{array}$ & $\begin{array}{c}\text { Macroobject } \\
\text { Radius } R_{\min }, \mathrm{m}\end{array}$ & $\begin{array}{c}\text { Macroobject } \\
\text { Density } \rho_{\max }, \mathrm{kg} \cdot \mathrm{m}^{-3}\end{array}$ \\
\hline DMF1 & $1.3 \times 10^{6}$ & $1.9 \times 10^{30}$ & $8.6 \times 10^{3}$ & $7.2 \times 10^{17}$ \\
DMF2 & $9.6 \times 10^{3}$ & $1.9 \times 10^{30}$ & $8.6 \times 10^{3}$ & $7.2 \times 10^{17}$ \\
Electron-Positron & 0.51 & $6.6 \times 10^{36}$ & $2.9 \times 10^{10}$ & $6.3 \times 10^{4}$ \\
DMF3 & $3.7 \times 10^{-3}$ & $1.2 \times 10^{41}$ & $5.4 \times 10^{14}$ & $1.8 \times 10^{-4}$ \\
DMF4 & $2 \times 10^{-7}$ & $4.2 \times 10^{49}$ & $1.9 \times 10^{23}$ & $1.5 \times 10^{-21}$ \\
\hline
\end{tabular}

- Nuclei made of self-annihilating DMF1 and/or DMF2 compose Cores of stars in extrasolar systems;

- Shells of electron-positron plasma around Nuclei made up of self-annihilating DMF1 and/or DMF2 make up Cores of globular clusters. Electron-positron plasma explains radio properties of Macroobjects;

- Shells of DMF3 around Nuclei made up of self-annihilating DMF1 and/or DMF2 with shells of electron-positron plasma make up Cores of galaxies;

- Nuclei made of DMF1 and/or DMF2 surrounded by shells of DMF3 and DMF4 compose Cores of superclusters.

Macroobject Cores have the following properties [1]:

- The minimum radius of Core $R_{\min }$ made of any fermion equals to three Schwarzschild radii;

- Core density does not depend on $M_{\max }$ and $R_{\min }$ and does not change in time while $M_{\max } \propto \tau^{3 / 2}$ and $R_{\min } \propto \tau^{1 / 2}$.

K. Mehrgan, et al. observed a supergiant elliptical galaxy Holmberg 15A about 700 million light-years from Earth. They found an Extreme Core with a mass of $4 \times 10^{10}$ solar masses at the center of Holm 15A [36]. The calculated maximum mass of galaxy Core of $6 \times 10^{10}$ solar masses (see Table 2) is in good agreement with the experimentally found value [36].

\subsection{The Beginning of the World}

Before the Beginning of the World, there was nothing but an Eternal Universe. About 14.2 billion years ago the World was started by a fluctuation in the Eternal Universe, and the Nucleus of the World, which is a four-dimensional ball, was born. An extrapolated Nucleus radius at the Beginning was equal to a basic unit of size $a$. The 3D World is a hypersphere that is the surface of a 4-ball Nucleus. All points of the hypersphere are equivalent; there are no preferred centers or boundary of the World [1]. Hypersphere World as a model of a finite universe was proposed by Georg Riemann in 1854 [37].

\subsection{Expansion and Creation of Matter}

The Nucleus is expanding in the Universe, and its surface, the hypersphere, is 
likewise expanding. The radius of the Nucleus $R$ is increasing with speed $c$ (gravitodynamic constant) for the absolute cosmological time $\tau$ from the Beginning and equals to $R=c \tau$. The expansion of the Hypersphere World can be understood through the analogy with an expanding 3D balloon: imagine an ant residing on a seemingly two-dimensional surface of a balloon. As the balloon is blown up, its radius increases, and its surface grows. The distance between any two points on the surface increases. The ant sees her world expand but does not observe a preferred center.

According to WUM, the surface of the Nucleus is created in a process analogous to sublimation. Continuous creation of matter is the result of such process. Sublimation is a well-known endothermic process that happens when surfaces are intrinsically more energetically favorable than the bulk of a material, and hence there is a driving force for surfaces to be created.

Matter comes from the Universe to the Nucleus along the fourth spatial dimension, passing through the 4-ball surface, which is our World. Dark Matter Particles (DMPs) carry new Matter into the Nucleus. By analogy with a three-dimensional ball, which has a two-dimensional sphere surface (that has surface energy), we can imagine that our three-dimensional World (Hypersphere) has a "Surface energy" of the four-dimensional Nucleus [1].

It is important to emphasize that

- Creation of Matter is a direct consequence of expansion;

- Creation of DM occurs homogeneously in all points of the hypersphere World;

- Ordinary Matter is a byproduct of DM annihilation. Consequently, the matter-antimatter asymmetry problem discussed in literature does not arise (since antimatter does not get created by DM annihilation).

\subsection{Content of the World}

The existence of the Medium is a principal point of WUM. It follows from the observations of Intergalactic Plasma; Cosmic Microwave Background Radiation (MBR); Far-Infrared Background Radiation. Inter-galactic voids discussed by astronomers are in fact examples of the Medium in its purest. Cosmic MBR is part of the Medium; it then follows that the Medium is the absolute frame of reference. Relative to MBR rest frame, Milky Way galaxy and Sun are moving with the speed of 552 and $370 \mathrm{~km} / \mathrm{s}$ respectively [1].

The Medium consists of stable elementary particles with lifetimes longer than the age of the World: DMPs in the Dark Epoch and protons, electrons, photons, neutrinos, and DMPs in the Luminous Epoch (see Sections 3.7, 3.8 and 3.9). For all particles under consideration we use the following characteristics:

- Type of particle (fermion or boson);

- "Mass" that is equivalent to "Rest energy" with the constant $c^{2}$;

- Electrical charge.

The total energy density of the Medium is $2 / 3$ of the overall energy density 
of the World. Superclusters, Galaxies, Extrasolar systems, planets, moons, etc. are made of the same particles. The energy density of Macroobjects adds up to $1 / 3$ of the total energy density of the World throughout the World's evolution [1].

In WUM, Time and Space are closely connected with Mediums' impedance and gravitomagnetic parameter. It follows that neither Time nor Space could be discussed in absence of the Medium. The gravitational parameter $G$ that is proportional to the Mediums' energy density can be introduced only for the Medium filled with Matter. In frames of WUM, the Gravitation is a result of simple interactions of DMF4 with Matter that work cooperatively to create a more complex interaction. DMF4 particles are responsible for the Le Sage's mechanism of the gravitation [1].

As a conclusion, Gravity, Space and Time are all emergent phenomena [1]. In this regard, it is worth to recall the Albert Einstein quote: "When forced to summarize the theory of relativity in one sentence: time and space and gravitation have no separate existence from matter".

\subsection{Inter-Connectivity of Primary Cosmological Parameters}

The constancy of the universe fundamental constants, including Newtonian constant of gravitation and Planck mass, is now commonly accepted, although has never been firmly established as a fact. All conclusions on the (almost) constancy of the Newtonian parameter of gravitation are model-dependent.

A commonly held opinion states that gravity has no established relation to other fundamental forces, so it does not appear possible to calculate it from other constants that can be measured more accurately, as is done in some other areas of physics. WUM holds that there indeed exist relations between all primary cosmological parameters that depend on dimensionless time-varying quantity $Q$ : Newtonian parameter of gravitation; Hubble's parameter; Age of the World; the Worlds' radius of curvature in the fourth spatial dimension; Critical energy density; Concentration of Intergalactic Plasma; Minimum Energy of Photons; Temperature of the Microwave Background Radiation; Temperature of the Far-Infrared Background Radiation peak [1].

Comparison of the calculated cosmological parameters based on the average value of the gravitational parameter with experimentally measured parameters is presented in Table 3.

Note that the precision of the most parameters value has increased by three orders of magnitude. We are not aware of any other model that allows calculation of CMB temperature with such accuracy [1].

The remarkable agreement of the calculated values of the primary cosmological parameters with the observational data gives us considerable confidence in the Model. We propose to introduce $Q$ as a new Fundamental Parameter tracked by CODATA and use its value in calculation of all $Q$-dependent parameters [1]. 
Table 3. Theoretical and experimental values of the primary cosmological parameters.

\begin{tabular}{|c|c|c|c|}
\hline Parameter & Theory & Experiment & Ref. \\
\hline \multirow[t]{3}{*}{ Hubble’s Parameter, km/s Mpc } & & $69.32 \pm 0.8$ & [38] \\
\hline & 68.7457 & $69.1_{-0.6}^{+0.4}$ & [39] \\
\hline & & $69.8 \pm 1.9$ & [40] \\
\hline CMB Temperature, $\mathrm{K}$ & 2.72518 & $2.72548 \pm 0.00057$ & [41] \\
\hline Concentration of Intergalactic Plasma, $\mathrm{m}^{-3}$ & 0.25480 & $\lesssim 0.27$ & [42] \\
\hline Proton Relative Energy Density & 0.048014655 & $0.049 \pm 0.013$ & [8] \\
\hline Minimum Energy of Photons, $\times 10^{-14} \mathrm{eV}$ & 1.8743 & $\$ 2.2$ & [43] \\
\hline Far-Infrared Background Peak, K & 28.955 & 29 & [44] \\
\hline
\end{tabular}

\subsection{Dark Epoch}

As we mentioned in Introduction, the Angular Momentum problem is one of the most critical problems in any Cosmological model that must be solved. To be consistent with the Law of Conservation of Angular Momentum a Model must answer the following questions:

- How did Galaxies and Extra Solar systems obtain their substantial orbital and rotational angular momenta;

- Why are all Macroobjects rotating;

- How did Milky Way give birth to different Extra Solar systems at different times;

- The beginning of the Milky Way (MW) galaxy was about 13.8 billion years. The age of MW is about the Age of the World. What is the origin of MW huge angular momentum? We must discuss the Beginning of the MW;

- The beginning of the Solar System (SS) was 4.6 billion years. What is the origin of SS angular momentum? We must discuss the Beginning of the SS;

- Why is the orbital momentum of Jupiter much larger than rotational momentum of the Sun. There is no possible means by which the angular momentum from the Sun could be transferred to the planets;

- In the theory of planetary formation, all planets, being made of the same ingredients, should have the same composition, yet they don't.

In our opinion, there is the only one mechanism that can provide angular momenta to Macroobjects-Rotational Fission of overspinning Prime Objects. From the point of view of Fission model, the prime object is transferring some of its rotational angular momentum to orbital and rotational momenta of satellites. It follows that the rotational momentum of the prime object should exceed the orbital momentum of its satellite. In frames of WUM, Prime Objects are DM Cores of Superclusters, which must accumulate tremendous angular momenta before the Birth of the Luminous World. It means that it must be some long enough time in the history of the World, which we named "Dark Epoch" [1].

Dark Epoch started at the beginning of the World and lasted for about 0.4 bil- 
lion years. WUM is a classical model, therefore classical notions can be introduced only when the very first ensemble of particles was created at the cosmological time $\cong 10^{-18} \mathrm{~s}$. At time $\tau \gg 10^{-18} \mathrm{~s}$ density fluctuations could happen in the Medium of the World filled with DMPs: DMF1, DMF2, DIRACs, ELOPs, DMF3 and DMF4. The heaviest Dark Matter particles DMF1 could collect into a cloud with distances between particles smaller than the range of the weak interaction $R_{W}$. As a result of the weak interaction, clumps of DMF1 will arise. Larger clumps will attract smaller clumps of DMPs and initiate a process of expanding the DM clump followed by growth of surrounding shells made up of other DMPs, up to the maximum mass of the shell made up of DMF4 at the end of Dark Epoch (0.4 billion years).

The process described above is the formation of the DM Core of a Supercluster. DMPs supply not only additional mass $\left(\propto \tau^{3 / 2}\right)$ to Cores, but also additional angular momentum $\left(\propto \tau^{2}\right)$ fueling the overspinning of Dark Matter Cores. In our opinion, all Supercluster Cores had undergone rotational fission at approximately the same cosmological time [1].

\subsection{Rotational Fission}

According to WUM, the rotational angular momentum of overspinning (surface speed at equator exceeding escape velocity) objects before rotational fission equals to [1]:

$$
L_{\text {rot }}=\frac{4 \sqrt{2}}{15} \frac{1+5 \delta}{1+3 \delta} G^{0.5} M^{1.5} R^{0.5}
$$

where $M$ is a mass of overspinning object, $R$ is its radius, $\delta$ is the density ratio inside of the object: $\delta=\rho_{\min } / \rho_{\max }$. Parameters $G, M, R$ for Macroobjects Cores are time-varying: $G \propto \tau^{-1}, M \propto \tau^{3 / 2}$ and $R \propto \tau^{1 / 2}$. It follows that the rotational angular momentum of Cores $L_{r o t}$ is proportional to $\tau^{2}$.

Local Supercluster (LS) is a mass concentration of galaxies containing the Local Group, which in turn contains the MW galaxy. At least 100 galaxy groups and clusters are located within its diameter of $1.1 \times 10^{8}$ light-years. Considering parameters of DMF4 shell (see Table 2), we calculate the rotational angular momentum $L_{r o t}^{L S C}$ of LS Core before rotational fission:

$$
L_{\text {rot }}^{L S C}=3.7 \times 10^{77} \mathrm{~J} \cdot \mathrm{S}
$$

MW is gravitationally bounded with LS [13]. Let's compare $L_{\text {rot }}^{L S C}$ with an orbital momentum of Milky Way $L_{o r b}^{M W}$ calculated based on the distance of 65 million light-years from LS Core and orbital speed of about $400 \mathrm{~km} / \mathrm{s}$ [13]:

$$
L_{\text {orb }}^{M W}=2.5 \times 10^{71} \mathrm{~J} \cdot \mathrm{S}
$$

It means that as the result of rotational fission of LS Core, approximately $\sim 10^{6}$ galaxies like Milky Way could be generated at the same time. Considering that density of galaxies in the LS falls off with the square of the distance from its center near the Virgo Cluster, and the location of MW on the outskirts of the LS 
[45], the actual number of created galaxies could be much larger.

The mass-to-light ratio of the LS is about 300 times larger than that of the Solar ratio. Similar ratios are obtained for other superclusters [46]. These facts support the rotational fission mechanism proposed above. In 1933, Fritz Zwicky investigated the velocity dispersion of Coma cluster and found a surprisingly high mass-to-light ratio ( 500). He concluded: if this would be confirmed, we would get the surprising result that dark matter is present in much greater amount than luminous matter [47]. These ratios are one of the main arguments in favor of presence of large amounts of Dark Matter in the World.

Analogous calculations for MW Core based on parameters of DMF3 shell (see Table 2) produce the following value of rotational angular momentum $L_{r o t}^{M W C}$ [1]:

$$
L_{r o t}^{M W C}=2.4 \times 10^{60} \mathrm{~J} \cdot \mathrm{s}
$$

which far exceeds the orbital momentum of the Solar System $L_{\text {orb }}^{S S} \quad$ calculated based on the distance from the galactic center of $26.4 \mathrm{kly}$ and orbital speed of about $220 \mathrm{~km} / \mathrm{s}$

$$
L_{\text {orb }}^{S S}=1.1 \times 10^{56} \mathrm{~J} \cdot \mathrm{S}
$$

As the result of rotational fission of MW Core 13.8 billion years ago, approximately $\sim 10^{4}$ Extrasolar systems like Solar System could be created at the same time. Considering that MW has grown inside out (in the present Epoch, most old stars can be found in the middle, more recently formed ones on the outskirts [48]), the number of generated Extrasolar systems could be much larger. Extrasolar system Cores can give birth to planetary cores, which in turn can generate cores of moons by the same Rotational Fission mechanism [1].

The oldest known star HD 140283 (Methuselah star) is a subgiant star about 190 light-years away from Earth for which a reliable age has been determined [49]. H. E. Bond, et al. found its age to be $14.46 \pm 0.8 \mathrm{Gyr}$ that does not conflict with the age of the Universe, $13.77 \pm 0.06 \mathrm{Gyr}$, based on the microwave background and Hubble constant [49]. It means that this star must have formed between 13.66 and $13.83 \mathrm{Gyr}$, amount of time that is too short for formation of the second generation of stars according to prevailing theories.

In WUM this discovery can be explained by generation of the Core of HD 140283 by overspinning Core of the MW 13.8 billion years ago.

In frames of the developed Rotational Fission model, it is easy to explain hyper-runaway stars unbound from the Milky Way with speeds of up to $\sim 700 \mathrm{~km} / \mathrm{s}$ [50]: they were launched by overspinning Core of the Large Magellan Cloud with the speed higher than the escape velocity [1].

S. E. Koposov, et al. present the discovery of the fastest Main Sequence hyper-velocity star S5-HVS1 with mass about 2.3 solar masses that is located a distance of $\sim 9 \mathrm{kpc}$ from the Sun. When integrated backwards in time, the orbit of the star points unambiguously to the Galactic Centre, implying that S5-HVS1 was kicked away from Sgr $A^{\star}$ with a velocity of $\sim 1800 \mathrm{~km} / \mathrm{s}$ and travelled for 4.8 
Myr to the current location. So far, this is the only hyper-velocity star confidently associated with the Galactic Centre [51].

In frames of the developed Model this discovery can be explained by Gravitational Burst of the overspinning Core of the Milky Way 4.8 million years ago, which gave birth to S5-HVS1 with the speed higher than the escape velocity of the Core (see Section 3.9).

A. Irrgang, et al. present the discovery of a new extreme runaway star, PG $1610+062$ challenging classical ejection mechanisms [52]. A kinematic analysis, based on the spectrophotometric distance $(17.3 \mathrm{kpc})$ shows that PG $1610+062$ was shot into the halo $\sim 41 \mathrm{Myr}$ ago from a region with a Galactocentric radius of $\sim 6.5 \mathrm{kpc}$, which possibly coincided with the location of the now nearby Carina-Sagittarius spiral arm at a velocity of $550 \pm 40 \mathrm{~km} / \mathrm{s}$ which is beyond the classical limits [52].

In frames of WUM, this discovery can be explained by a Gravitational Burst of the overspinning Core of the globular cluster M55 in the constellation Sagittarius. M55 is at a distance of about 17.6 kly away from Earth. It has a mass of about $2.69 \times 10^{5}$ times that of the Sun and estimated age $12.3 \mathrm{Gyr}$.

C. J. Clarke, et al. observed CI Tau, a young 2 million years old star. CI Tau is located about 500 light-years away in a highly-productive stellar 'nursery' region of the galaxy. They discovered that the Extrasolar System contains four gas giant planets that are only 2 million years old [53], amount of time that is too short for formation of gas giants according to prevailing theories.

In frames of the developed Rotational Fission model, this discovery can be explained by Gravitational Burst of the overspinning Core of the Milky Way two million years ago, which gave birth to CI Tau system with DM cores of all planets generated at the same time.

To summarize,

- The rotational fission of macroobject Cores is the most probable process that can generate satellite cores with large orbital and rotational momenta in very short time;

- Macrostructures of the World form from the top (superclusters) down to galaxies, extrasolar systems, planets, and moons;

- Gravitational waves can be a product of rotational fission of overspinning Macroobject Cores;

- The Big Bang discussed in the Standard cosmological model is, in our view, the transition from Dark Epoch to Luminous Epoch.

\subsection{Luminous Epoch}

Luminous Epoch spans from 0.4 billion years up to the present Epoch (during 13.8 billion years). According to WUM, Cores of all Macroobjects (MOs) of the World (Superclusters, Galaxies, Extrasolar systems) possess the following properties [1]:

- Their Nuclei are made up of DMFs and contain other particles, including 
Dark Matter and Ordinary matter, in shells surrounding the Nuclei;

- DMPs are continuously absorbing by Cores of all MOs. Ordinary Matter (about $7.2 \%$ of the total Matter in the World) is a byproduct of DMPs annihilation. It is re-emitted by Cores of MOs continuously;

- Nuclei and shells are growing in time: size $\propto \tau^{1 / 2}$; mass $\propto \tau^{3 / 2}$; and rotational angular momentum $\propto \tau^{2}$, until they reach the critical point of their stability, at which they detonate. Satellite cores and their orbital $L_{o r b}$ and rotational $L_{r o t}$ angular momenta released during detonation are produced by Overspinning Core (OC). The detonation process does not destroy OC; it's rather gravitational hyper-flares;

- Size, mass, composition, $L_{o r b}$ and $L_{r o t}$ of satellite cores depend on local density fluctuations at the edge of OC and cohesion of the outer shell. Consequently, the diversity of satellite cores and their rotation have a clear explanation.

WUM refers to OC detonation process as Gravitational Burst (GB), analogous

to Gamma Ray Burst. In frames of WUM, the repeating GBs can be explained the following way:

- As the result of GB, the OC loses a small fraction of its mass and a large part of its rotational angular momentum;

- After GB, the Core absorbs new DMPs. Its mass increases $\propto \tau^{3 / 2}$, and its angular momentum $L_{\text {rot }}$ increases much faster $\propto \tau^{2}$, until it detonates again at the next critical point of its stability. Consequently, the birth to different Extra Solar systems at different times has a clear explanation;

- Afterglow of GBs is a result of processes developing in the Nuclei and shells after detonation;

- In case of Extrasolar systems, a star wind is the afterglow of star detonation: star Core absorbs new DMPs, increases its mass $\propto \tau^{3 / 2}$ and gets rid of extra $L_{\text {rot }}$ by star wind particles;

- Solar wind is the afterglow of Solar Core detonation 4.6 billion years ago. It creates the bubble of the Heliosphere continuously;

- In case of Galaxies, a galactic wind is the afterglow of repeating galactic Core detonations. In Milky Way, it continuously creates two Dark Matter Fermi Bubbles.

\subsection{Dark Matter Fermi Bubbles}

In November 2010, the discovery of two Fermi Bubbles (FBs) emitting gammaand X-rays was announced. FBs extend for about $25 \mathrm{kly}$ above and below the center of the galaxy [54]. The outlines of the bubbles are quite sharp, and the bubbles themselves glow in nearly uniform gamma rays over their colossal surfaces. Gamma-ray spectrum measured by the Fermi Large Area Telescope remains unconstrained up to around $1 \mathrm{TeV}$ [55]. Years after the discovery of FBs, their origin and the nature of the gamma-ray emission remain unresolved.

M. Su and D. P. Finkbeiner [56] identify a gamma-ray cocoon feature in the 
southern Fermi bubble, a jet-like feature along the cocoon's axis of symmetry, and another directly opposite the Galactic center in the north. Both the cocoon and jet-like feature have a hard spectrum from 1 to $100 \mathrm{GeV}$. If confirmed, these jets are the first resolved gamma-ray jets ever seen.

G. Ponti, et al. report prominent X-ray structures on intermediate scales (hundreds of parsecs) above and below the plane, which appear to connect the Galactic Centre region to the Fermi bubbles. They propose that these structures, which they term the Galactic Centre "chimneys", constitute exhaust channels through which energy and mass, injected by a quasi-continuous train of episodic events at the Galactic Centre, are transported from the central few parsecs to the base of the FBs [57].

D. Hooper and T. R. Slatyer discuss two emission mechanisms in the FBs: inverse Compton scattering and annihilating DM [58]. In their opinion, the second emission mechanism must be responsible for the bulk of the low-energy, low-latitude emission. The spectrum and angular distribution of the signal is consistent with that predicted from $\sim 10 \mathrm{GeV}$ DMPs annihilating to leptons. This component is similar to the excess $\mathrm{GeV}$ emission previously reported by $\mathrm{D}$. Hooper from the Galactic Center [59].

It is worth to note that a similar excess of gamma-rays was observed in the central region of the Andromeda galaxy (M31). A. McDaniel, T. Jeltema, and S. Profumo calculated the expected emission across the electromagnetic spectrum in comparison with available observational data from M31 and found that the best fitting models are with the DMP mass $11 \mathrm{GeV}[60]$.

In frames of WUM, the Galactic Center Gamma-Ray Excess at energy about $10 \mathrm{GeV}$ is consistent with the annihilation of the particles DMF2 with mass 9.6 $\mathrm{GeV}$.

According to H.-Y. K. Yang, M. Ruszkowski, and E. G. Zweibel, for understanding the physical origin of the FBs, three major questions need to be answered [61]:

- First, what is the emission mechanism? The bubbles can either be hadronic, where the gamma rays are produced by inelastic collisions between cosmic-ray protons and the thermal nuclei via decay of neutral pions, or leptonic, where the gamma rays are generated by inverse-Compton scattering of the interstellar radiation field by cosmic-ray electrons;

- Second, what activity at the Galactic Center triggered the event-are the bubble associated with nuclear star formation or active galactic nucleus activity?

- Third, where are the Cosmic rays accelerated? They could either be accelerated at the Galactic Center and transported to the surface of the bubbles or accelerated in-situ by shocks or turbulence. Note however that not all combinations of the above three considerations would make a successful model because of constraints given by the hard spectrum of the observed bubbles.

WUM explains FBs the following way [1]:

- Core of MW galaxy is made up of DM particles: DMF1 (1.3 TeV), DMF2 (9.6 
$\mathrm{GeV}$ ), and DMF3 (3.7 keV). The second component (DMF2) explains the excess $\mathrm{GeV}$ emission reported by Dan Hooper from the Galactic Center [59]. Core rotates with surface speed at equator close to the escape velocity between Gravitational Bursts (GBs), and over the escape velocity at the moments of GBs;

- Bipolar astrophysical jets (which are astronomical phenomena where outflows of matter are emitted as an extended beams along the axis of rotation [62]) of DMPs are ejected from the rotating Core into the Galactic halo along the rotation axis of the Galaxy;

- Due to self-annihilation of DMF1 and DMF2, these beams are gamma-ray jets [56];

- The prominent X-ray structures on intermediate scales (hundreds of parsecs) above and below the plane (named the Galactic Centre "chimneys" [57]) are the result of the self-annihilation of DMF3;

- FBs are bubbles with boundary between them and Intergalactic Medium that has a surface energy density equals to the basic unit of surface energy density $\sigma_{0}=h c / a^{3}$;

- These bubbles are filled with DM particles: DMF1, DMF2, and DMF3. In our Model, FBs are Macroobjects with a mass $M_{F B}$ and diameter $D_{F B}$, which are proportional to: $M_{F B} \propto Q^{3 / 2}$ and $D_{F B} \propto Q^{3 / 4}$ respectively. According to WUM, diameter of FBs equals to:

$$
D_{F B}=L_{\mathrm{DMF} 3} \times Q^{3 / 4}=\frac{a}{\alpha^{2}} \times Q^{3 / 4}=28.6 \mathrm{kly}
$$

where $L_{\mathrm{DMF} 3}$ is Compton length of particles DMF3 with mass $m_{\mathrm{DMF} 3}=\alpha^{2} m_{0}$. The calculated diameter is in good agreement with the measured size of the FBs $25 \mathrm{kly}$ [53] and $32.6 \mathrm{kly}$ [55];

- With Nikola Tesla's principle at heart-There is no energy in matter other than that received from the environment-we calculate mass $M_{F B}$ :

$$
M_{F B}=\frac{\pi D_{F B}^{2} \sigma_{0}}{c^{2}}=\frac{\pi m_{0}}{\alpha^{4}} \times Q^{3 / 2} \cong 3.6 \times 10^{41} \mathrm{~kg}
$$

Recall that the mass of Milky Way galaxy $M_{M W}$ is about: $M_{M W} \cong 3.2 \times 10^{42} \mathrm{~kg}$;

- Weak interaction between DMF3 particles provides integrity of Fermi Bubbles. FBs made up of DMF3 particles resembles a honeycomb filled with DMF1 and DMF2;

- FBs radiate X-rays due to the annihilation of DMF3 particles;

- Gamma rays up to $1 \mathrm{TeV}$ [52] are the result of annihilation of DMF1 (1.3 TeV) and DMF2 (9.6 GeV) in Dark Matter Objects (DMOs) whose density is sufficient for the annihilation of DMPs to occur. DMOs are much smaller than stars in the World, have a high concentration in FBs and provide nearly uniform gamma ray glow over their colossal surfaces. The total flux of the gamma radiation from $\mathrm{FBs}$ is the sum of the contributions of all individual DMOs; 
- The Core of the Milky Way supplies FBs with new DMPs through the galactic wind, explaining the brightness of FBs remaining fairly constant during the time of observations. FBs are built continuously throughout the lifetime of Milky Way (13.8 By).

In our view, FBs are DMP clouds containing uniformly distributed clumps of Dark Matter Objects, in which DMPs annihilate and radiate X-rays and gamma rays. Dark Matter Fermi Bubbles constitute a principal proof of the World Universe Model.

\subsection{Hypersphere World}

The physical laws we observe appear to be independent of the Worlds' curvature in the fourth spatial dimension due to the very small value of the dimension-transposing gravitomagnetic parameter of the Medium [1]. Consequently, direct observation of the Worlds' curvature would appear to be a hopeless goal.

One way to prove the existence of the Worlds' curvature is direct measurement of truly large-scale parameters of the World: Gravitational, Hubble's, Temperature of the Microwave Background Radiation. Conducted at various points of time, these measurements would give us varying results, providing insight into the curved nature of the World. Unfortunately, the accuracy of the measurements is quite poor. Measurement errors far outweigh any possible "curvature effects", rendering this technique useless in practice. To be conclusive, the measurements would have to be conducted billions of years apart.

In WUM, Local Physics is linked with the large-scale structure of the Hypersphere World through the dimensionless quantity $Q$. The proposed approach to the fourth spatial dimension agrees with Mach's principle: "Local physical laws are determined by the large-scale structure of the universe". Applied to WUM, it follows that all parameters of the World depending on $Q$ are a manifestation of the Worlds' curvature in the fourth spatial dimension.

\section{Conclusions}

In conclusion, we postulate the principal role of Angular Momentum and Dark Matter in Cosmological theories of the World.

Dark Matter is abundant:

- $2.4 \%$ of Ordinary Matter is in Superclusters, Galaxies, Stars, Planets, etc.

- $4.8 \%$ of Ordinary Matter is in the Medium of the World;

- The remaining $92.8 \%$ of mass is Dark Matter;

- WUM predicts existence of DM particles with $1.3 \mathrm{TeV}, 9.6 \mathrm{GeV}, 70 \mathrm{MeV}$, $340 \mathrm{keV}, 3.7 \mathrm{keV}$, and $0.2 \mathrm{eV}$ masses.

Based on the totality of the results obtained by WUM [1], we suggest adopting existence of Dark Matter in the World from the Classical Physics point of view.

To be consistent with the Law of Conservation of Angular Momentum we develop New Physics of the World:

- The Model introduces Dark Epoch (spanning from the Beginning of the World 
for 0.4 billion years) when only Dark Matter Particles existed, and Luminous Epoch (ever since for 13.8 billion years) when Dark Matter and Ordinary Matter exist;

- The main players of the World are overspinning DM Cores of Superclusters, which accumulated tremendous rotational angular momenta during Dark Epoch and transferred it to DM Cores of Galaxies during their Rotational Fission;

- Big Bang discussed in Standard Cosmology is a transition from Dark Epoch to Luminous Epoch due to Rotational Fission of Overspinning DM Supercluster's Cores;

- Dark Matter Core of Milky Way galaxy was born 13.8 billion years ago as the result of the Rotational Fission of the Local Supercluster DM Core;

- DM Cores of Extrasolar systems, planets and moons were born as the result of Gravitational Bursts of the Milky Way DM Core at different times (4.6 billion years ago for the Solar system);

- Proposed Weak Interaction between DMPs provides the integrity of DM Cores of all Macroobjects.

The Hypersphere World-Universe Model successfully describes primary cosmological parameters and their relationships, ranging in scale from cosmological structures to elementary particles. WUM allows for precise calculation of their values that were only measured experimentally earlier and makes verifiable predictions.

WUM does not attempt to explain all available cosmological data, as that is an impossible feat for any one manuscript. Nor does WUM pretend to have built an all-encompassing theory that can be accepted as is. The Model needs significant further elaboration, but in its present shape, it can already serve as a basis for a new Physics proposed by Paul Dirac in 1937. The Model should be developed into the well-elaborated theory by all physical communities.

\section{Acknowledgements}

I am a Doctor of Sciences in Physics. I belong to the school of physicists established by Alexander Prokhorov-Nobel Prize Laureate in Physics. I am an author of more than 150 published papers, mostly in the area of Laser Physics. I am eternally grateful to Prof. A. M. Prokhorov and Prof. A. A. Manenkov, whose influence on my scientific life has been decisive.

For the past 18 years, I have been developing a model I dubbed the WorldUniverse Model and published a serious of papers in the Journal of High Energy Physics, Gravitation and Cosmology (JHEPGC). I am much obliged to Prof. C. Corda for publishing my manuscripts in JHEPGC.

Many thanks to my long-term friend Felix Lev for stimulating discussions of history and philosophy of Physics and important comments on the Model. Special thanks to my son Ilya Netchitailo who questioned every aspect of the Model, gave valuable suggestions and helped shape it to its present form. 


\section{Conflicts of Interest}

The author declares no conflicts of interest regarding the publication of this paper.

\section{References}

[1] Netchitailo, V. (2019) Dark Matter Cosmology and Astrophysics. Journal of High Energy Physics, Gravitation and Cosmology, 5, 999-1050. https://doi.org/10.4236/jhepgc.2019.54056

[2] The Four Pillars of the Standard Cosmology. http://www.damtp.cam.ac.uk/research/gr/public/bb_pillars.html

[3] Shortcomings of the Standard Cosmology. http://www.damtp.cam.ac.uk/research/gr/public/bb_problems.html

[4] Couronne, I. and Ahmed, I. (2019) Top Cosmologist's Lonely Battle against "Big Bang” Theory. https://phys.org/news/2019-11-cosmologist-lonely-big-theory.html

[5] Silk, J. (2018) Towards the Limits of Cosmology. Foundations of Physics, 48, 1305-1332. https://doi.org/10.1007/s10701-018-0183-y

[6] Conover, E. (2019) Debate over the Universe's Expansion Rate May Unravel Physics. Is It a Crisis? ScienceNews.

https://www.sciencenews.org/article/debate-universe-expansion-rate-hubble-consta nt-physics-crisis

[7] Verde, L., Treu, T. and Riess, A.G. (2019) Tensions between the Early and the Late Universe. Nature Astronomy, 3, 891-895.

https://doi.org/10.1038/s41550-019-0902-0

[8] Keane, E.F., et al. (2016) A Fast Radio Burst Host Galaxy. Nature, 530, 453-456. https://doi.org/10.1038/nature17140

[9] Wikipedia. Big Bang Nucleosynthesis. https://en.wikipedia.org/wiki/Big_Bang_nucleosynthesis\#cite_ref-13

[10] Anders, M., et al. (2014) First Direct Measurement of the ${ }^{2} \mathrm{H}(\alpha, \gamma){ }^{6} \mathrm{Li}$ Cross Section at Big Bang Energies and the Primordial Lithium Problem. Physical Review Letters, 113, Article ID: 042501. https://doi.org/10.1103/PhysRevLett.113.042501

[11] Burbidge, E.M., Burbidge, G.R., Fowler, W.A. and Hoyle, F. (1957) Synthesis of the Elements in Stars. Reviews of Modern Physics, 29, 547-650.

https://doi.org/10.1103/RevModPhys.29.547

[12] Lopez-Corredoira, M. (2017) Tests and Problems of the Standard Model in Cosmology. Foundations of Physics, 47, 711-768.

https://doi.org/10.20944/preprints201702.0002.v1

[13] NASA (2015) The Cosmic Distance Scale. https://imagine.gsfc.nasa.gov/features/cosmic/local_supercluster_info.html

[14] Karachentsev, I. (1987) Double Galaxies. 7.1. The Orbital and Internal Angular Momentum of Galaxies in Pairs. Izdatel'stvo Nauka. Moscow. https://ned.ipac.caltech.edu/level5/Sept02/Keel/Keel7.html

[15] Toth, V.T. (2019) Is a Black Hole Technically 2-Dimensional? Quora. https://mail.google.com/mail/u/0/?tab=rm\&ogbl\#inbox/FMfcgxwGBmrfKchQCdbT TjWvmLcpPCpl

[16] Cahill, D. (2014) Radio Galaxy Discovery near Earth Spurs More Questions. https://phys.org/news/2014-05-radio-galaxy-discovery-earth-spurs.html

[17] Van Dokkum, P., et al. (2019) A High Stellar Velocity Dispersion and 100 Globu- 
lar Clusters for the Ultra Diffuse Galaxy Dragonfly 44. The Astrophysical Journal Letters, 828, L6. https://doi.org/10.3847/2041-8205/828/1/L6

[18] Narayan, R., McClintock, J.E. and Yi, I. (1995) A New Model for Black Hole Soft $\mathrm{X}$-Ray Transients in Quiescence. Astrophysical Journal, 457, 821. https://doi.org/10.1086/176777

[19] Gebhardt, K., Rich, R.M. and Luis Ho, L. (2002) A 20 Thousand Solar Mass Black Hole in the Stellar Cluster G1. Astrophysical Journal, 578, L41-L46. https://doi.org/10.1086/342980

[20] Chomiuk, L., et al. (2013) A Radio-Selected Black Hole X-Ray Binary Candidate in the Milky Way Globular Cluster M62. The Astrophysical Journal, 777, 69. https://doi.org/10.1088/0004-637X/777/1/69

[21] Giesers, B., et al. (2018) A Detached Stellar-Mass Black Hole Candidate in the Globular Cluster NGC 3201. Monthly Notices of the Royal Astronomical Society: Letters, 475, L15-L19. https://doi.org/10.1093/mnrasl/slx203

[22] Liu, J., et al. (2019) A Wide Star-Black-Hole Binary System from Radial-Velocity Measurements. Nature, 575, 618-621. https://doi.org/10.1038/s41586-019-1766-2

[23] Mersini-Houghton, L. (2014) Back-Reaction of the Hawking Radiation Flux on a Gravitationally Collapsing Star II. Physics Letters B, 738, 61-67. https://doi.org/10.1016/j.physletb.2014.09.018

[24] Leane, R.K. and Slatyer, T.R. (2019) Revival of the Dark Matter Hypothesis for the Galactic Center Gamma-Ray Excess. Physical Review Letters, 123, Article ID: 241101. https://doi.org/10.1103/PhysRevLett.123.241101

[25] Spolyar, D., Freese, K. and Gondolo, P. (2007) The Effect of Dark Matter and the First Stars: A New Phase of Stellar Evolution. AIP Conference Proceedings, 990, 42. https://doi.org/10.1063/1.2905656

[26] Freese, K., Rindler-Daller, T., Spolyar, D. and Valluri, M. (2015) Dark Stars: A Review. Reports on Progress in Physics, 79, Article ID: 066902. https://doi.org/10.1088/0034-4885/79/6/066902

[27] Lee, B.W. and Weinberg, S. (1977) Cosmological Lower Bound on Heavy-Neutrino Masses. Physical Review Letters, 39, 165-168. https://doi.org/10.1103/PhysRevLett.39.165

[28] Dicus, D.A., Kolb, E.W. and Teplitz, V.L. (1977) Cosmological Upper Bound on Heavy-Neutrino Lifetimes. Physical Review Letters, 39, 168-171. https://doi.org/10.1103/PhysRevLett.39.168

[29] Dicus, D.A., Kolb, E.W. and Teplitz, V.L. (1978) Cosmological Implications of Massive, Unstable Neutrinos. The Astrophysical Journal, 221, 327-341. https://doi.org/10.1086/156031

[30] Gunn, J.E., et al. (1978) Some Astrophysical Consequences of the Existence of a Heavy Stable Neutral Lepton. The Astrophysical Journal, 223, 1015-1031. https://doi.org/10.1086/156335

[31] Stecker, F.W. (1978) The Cosmic Gamma-Ray Background from the Annihilation of Primordial Stable Neutral Heavy Leptons. The Astrophysical Journal, 223, 1032-1036. https://doi.org/10.1086/156336

[32] Zeldovich, Ya.B., Klypin, A.A., Khlopov, M.Yu. and Chechetkin, V.M. (1980) Astrophysical Constraints on the Mass of Heavy Stable Neutral Leptons. Soviet Journal of Nuclear Physics, 31, 664-669.

[33] Corda, C. (2009) Interferometric Detection of Gravitational Waves: The Definitive Test for General Relativity. International Journal of Modern Physics, 18, 2275-2282. 
https://doi.org/10.1142/S0218271809015904

[34] Bertone, G. and Tait, T.M.P. (2018) A New Era in the Quest for Dark Matter.

[35] Boehm, C., Fayet, P. and Silk, J. (2003) Light and Heavy Dark Matter Particles. Physical Review D, 69, 101302(R). https://doi.org/10.1103/PhysRevD.69.101302

[36] Mehrgan, K., et al. (2019) A 40-Billion Solar Mass Black Hole in the Extreme Core of Holm 15A, the Central Galaxy of Abell 85. The Astrophysical Journal, 887, 195. https://doi.org/10.3847/1538-4357/ab5856

[37] Riemann, B. (1854) On the Hypotheses Which Lie at the Bases of Geometry. Nature, 8, 14-17, 36, 37. https://doi.org/10.1038/008036a0

[38] Bennett, C.L., et al. (2013) Nine-Year Wilkinson Microwave Anisotropy Probe (WMAP) Observations: Final Maps and Results. The Astrophysical Journal Supplement Series, 208, 20. https://doi.org/10.1088/0067-0049/208/2/20

[39] Abbott, T.M.C., et al. (2017) Dark Energy Survey Year 1 Results: A Precise H0 Measurement from DES Y1, BAO, and D/H Data. Monthly Notices of the Royal Astronomical Society, 480, 3879-3888. https://doi.org/10.1093/mnras/sty1939

[40] Freedman, W.L., et al. (2019) The Carnegie-Chicago Hubble Program. VIII. An Independent Determination of the Hubble Constant Based on the Tip of the Red Giant Branch. The Astrophysical Journal, 882, 34. https://doi.org/10.3847/1538-4357/ab2f73

[41] Fixsen, D.J. (2009) The Temperature of the Cosmic Microwave Background. The Astrophysical Journal, 707, 916. https://doi.org/10.1088/0004-637X/707/2/916

[42] Mirizzi, A., Raffelt, G.G. and Serpico, P.D. (2006) Photon-Axion Conversion in Intergalactic Magnetic Fields and Cosmological Consequences. Lecture Notes in Physics, 741, 115-134. https://doi.org/10.1007/978-3-540-73518-2_7

[43] Bonetti, L., et al. (2017) FRB 121102 Casts New Light on the Photon Mass. Physics Letters B, 768, 326-329. https://doi.org/10.1016/j.physletb.2017.03.014

[44] Lagache, G., et al. (1999) First Detection of the Warm Ionized Medium Dust Emission. Implication for the Cosmic Far-Infrared Background. Astronomy and Astrophysics, 344, 322-332.

[45] Tully, R.B. (1982) The Local Supercluster. Astrophysical Journal, 257, 389-422. https://doi.org/10.1086/159999

[46] Heymans, C., et al. (2008) The Dark Matter Environment of the Abell 901/902 Supercluster: A Weak Lensing Analysis of the HST STAGES Survey. Monthly Notices of the Royal Astronomical Society, 385, 1431-1442. https://doi.org/10.1111/j.1365-2966.2008.12919.x

[47] Zwicky, F. (1933) Die Rotverschiebung von extragalaktischen Nebeln. Helvetica Physica Acta, 6, 110-127.

[48] Ness, M., et al. (2015) The Cannon: A Data-Driven Approach to Stellar Label Determination. The Astrophysical Journal, 808, 16. https://doi.org/10.1088/0004-637X/808/1/16

[49] Bond, H.E., et al. (2013) HD 140283: A Star in the Solar Neighborhood That Formed Shortly after the Big Bang. The Astrophysical Journal Letters, 765, L12. https://doi.org/10.1088/2041-8205/765/1/L12

[50] Marchetti, T., Rossi, E.M. and Brown, A.G.A. (2018) Gaia DR2 in 6D: Searching for the Fastest Stars in the Galaxy. Monthly Notices of the Royal Astronomical Society, 490, 157-171. https://doi.org/10.1093/mnras/sty2592

[51] Koposov, S.E., et al. (2019) The Great Escape: Discovery of a Nearby 1700 km/s Star Ejected from the Milky Way by Sgr A*. Monthly Notices of the Royal Astronomical 
Society, 491, 2465-2480. https://doi.org/10.1093/mnras/stz3081

[52] Irrgang, A., et al. (2019) PG 1610 + 062: A Runaway B Star Challenging Classical Ejection Mechanisms. Astronomy \& Astrophysics, 628, L5.

https://doi.org/10.1051/0004-6361/201935429

[53] Clarke, C.J., et al. (2018) High-Resolution Millimeter Imaging of the CI Tau Protoplanetary Disk: A Massive Ensemble of Protoplanets from 0.1 to $100 \mathrm{au}$. The Astrophysical Journal Letters, 866, L6. https://doi.org/10.3847/2041-8213/aae36b

[54] Aguilar, D.A. and Pulliam, C. (2010) Astronomers Find Giant, Previously Unseen Structure in Our Galaxy. Harvard-Smithsonian Center for Astrophysics. Release No. 2010-22.

[55] Yang, L. and Razzaque, S. (2019) Constraints on Very High Energy Gamma-Ray Emission from the Fermi Bubbles with Future Ground-Based Experiments. Physical Review D, 99, Article ID: 083007. https://doi.org/10.1103/PhysRevD.99.083007

[56] Su, M. and Finkbeiner, D.P. (2012) Evidence for Gamma-Ray Jets in the Milky Way. The Astrophysical Journal, 753, 61. https://doi.org/10.1088/0004-637X/753/1/61

[57] Ponti, G., et al. (2019) An X-Ray Chimney Extending Hundreds of Parsecs above and below the Galactic Centre. Nature, 567, 347-350. https://doi.org/10.1038/s41586-019-1009-6

[58] Hooper, D. and Slatyer, T.R. (2013) Two Emission Mechanisms in the Fermi Bubbles: A Possible Signal of Annihilating Dark Matter. Physics of the Dark Universe, 2, 118-138. https://doi.org/10.1016/j.dark.2013.06.003

[59] Hooper, D. and Goodenough, L. (2011) Dark Matter Annihilation in the Galactic Center as Seen by the Fermi Gamma Ray Space Telescope. Physics Letters B, 697, 412-428. https://doi.org/10.1016/j.physletb.2011.02.029

[60] McDaniel, A., Jeltema, T. and Profumo, S. (2018) A Multi-Wavelength Analysis of Annihilating Dark Matter as the Origin of the Gamma-Ray Emission from M31. Physical Review D, 97, Article ID: 103021. https://doi.org/10.1103/PhysRevD.97.103021

[61] Yang, H.Y.K., Ruszkowski, M. and Zweibel, E.G. (2018) Unveiling the Origin of the Fermi Bubbles. Galaxies, 6, 29. https://doi.org/10.3390/galaxies6010029

[62] Beall, J.H. (2015) A Review of Astrophysical Jets. Proceedings of Science: 58. 